\title{
A Rotation Measure Gradient on the M87 VLA Jet
}

\author{
Juan Carlos Algaba ${ }^{1, a}$, Keiichi Asada ${ }^{1}$, and Masanori Nakamura ${ }^{1}$ \\ ${ }^{1}$ Academia Sinica, Institute of Astronomy and Astrophysics, 11F of Astronomy-Mathematics Building, AS/NTU No 1, Sec 4, \\ Roosevelt Rd., Taipei 10617, Taiwan
}

\begin{abstract}
Rotation measures (RMs) have proven to be an excellent tool to study magnetic field structures in AGNs. Here we study RM properties on kiloparsec scales of the M87 jet via stacked multi wavelength polarized VLA observations. Our results show for the first time an indication of the RM gradient transverse to the jet in knot A, and possibly knot C and HST-1. Motivated by the shape of the RM in knots A and B, we discuss that part of it may be a filamentary structure of higher RM due to an external Faraday screen, although we consider this unlikely The data presented here can be easily explained by a helical magnetic field. By combining this result together with polarization direction plus the shape and degree of the fractional polarization across the jet, we can fairly conclude the presence of systematically wrapped, possibly helical, magnetic fields tightly wounded in knots A and C, in agreement with an MHD quad shock model.
\end{abstract}

\section{Introduction}

The supergiant elliptical galaxy M87 hosting an active galactic nuclei (AGN) is thought to be driven by its activity via a central supermassive black hole (SMBH) with a mass of $M_{B H} \sim 3.2-6.6 \times 10^{9} M_{\odot}[1,2]$. It is one of the closest AGN with an extended radio-jet at a distance $\mathrm{D}_{L}=16.7 \mathrm{Mpc}$ [3], with an angular scale of $78 \mathrm{pc} \operatorname{arcsec}^{-1}$, and a viewing angle $\theta \sim 10^{\circ}-19^{\circ}$ constrained by superluminal motion and Doppler beaming factors $[4,5]$. Due to its proximity, and the possibility to spatially resolve its jet, M87 has been the model to compare with other more distant, unresolved AGN jets and has been intensively studied over decades.

High resolution images providing detailed morphology of the M87 jet have been extensively studied over thirty years in optical and radio bands [6-12]. Recent studies have shown that hydrodynamics alone cannot give rise to the rich structure both in total and polarized intensity and knots in the kilo parsec jet cannot be associated with Kelvin-Helmholtz instabilities [13]. Consequently, magnetic fields are needed in order to understand the complexity of the jet. However, the magnetic structure and its dynamical importance at kilo parsec scales are still under debate.

Several methods have been exploit in order to understand the structure of magnetic fields in AGN. The degree of polarization can provide us information about the homogeneity of magnetic fields and their relevance, whereas the polarization position angle provides us insight about the projected direction of the magnetic field. As rotation measure effects can give us hints about the line of sight component of the magnetic fields, once we combine these

\footnotetext{
a e-mail: algaba@asiaa.sinica.edu.tw
}

various information, we can infer the $3 \mathrm{D}$ magnetic configuration of the jet.

One way to study the structure of the intrinsic magnetic field in the jet is the analysis of Faraday rotation effects. The polarization angle $\chi$ rotates following the relation $\chi=\chi_{0}+\mathrm{RM} \lambda^{2}$, where $\chi_{0}$ is the intrinsic polarization angle, $\lambda$ the wavelength and RM the rotation measure, given by

$$
\mathrm{RM} \propto \int \mathrm{n}_{\mathrm{e}} \mathrm{B} \cdot \mathrm{dl}
$$

with $n_{e}$ the thermal electron density and $B \cdot d l$ the magnetic field along the line of sight. If the jet contains a helical magnetic field, its toroidal component will produce a rotation measure change across the jet, giving rise to a gradient [14]. Such gradients have been observed on parsec scales or single knots on kilo parsec scales of a variety of sources [15-22].

Polarization angles, once corrected for Faraday rotation, can also provide valuable information about the magnetic structure in the jet. Polarization vectors seem to follow the direction of the jet, even with considerable blending in several BL Lac objects [23-25]. Spine-sheath structure, where polarization seems to be parallel to the direction of the jet in the center and perpendicular to it as we move towards the edges, has been found in various sources [26]. Although initially interpreted as interaction with the surrounding media, this structure arises naturally with the presence of a helical magnetic field [23]. Furthermore, observations missing this structure do no imply the absence of helical magnetic field, as a combination of pitch and viewing angles may give rise to diverse observed polarization structures $[27,28]$.

If we study the fractional polarization across the jet, an increase towards the edges, asymmetric with respect to the 
Table 1. Observing Sessions.

\begin{tabular}{cccc}
\hline $\begin{array}{c}\text { Obs. Code } \\
(1)\end{array}$ & $\begin{array}{c}\text { Freq. (GHz) } \\
(2)\end{array}$ & $\begin{array}{c}\text { VLA Conf. } \\
(3)\end{array}$ & $\begin{array}{c}\text { Date } \\
(4)\end{array}$ \\
\hline AH822A & $8,15,22$ & A & 2 Jun 2003 \\
AH822B & $8,15,22$ & A & 24 Aug 2003 \\
AH822C & $15,22,43$ & B & 16 Nov 2003 \\
AH862A & $8,15,22$ & A & 15 Nov 2004 \\
AH862B & $8,15,22$ & A & 31 Dec 2004 \\
AH862C & $15,22,43$ & B & 3 May 2005 \\
\hline
\end{tabular}

jet axis, is expected if we assume a helical magnetic field with a certain viewing angle $[18,29,30]$. It is also possible to have a similar scenario if a shock were a random magnetic field is compressed in a plane [31]. However, asymmetries in this case are hard to be modeled except for very restricted scenarios and an intrinsically weak, random magnetic field may not be in agreement with observations showing a high degree of polarization [32].

\section{Archival Data Reduction}

We aim to use all these methods in order to understand the magnetic field structure on the kilo parsec scales of the M87 jet. In order to do so, we use the image stacking technique, which has proven to be a very powerful tool in order to enhance sensitivity and reveal features that otherwise were hidden or difficult to disentangle from the noise $[18,33,34]$. We examined six different epochs of VLA multi-frequency polarization observations of M87 obtained from the $\operatorname{archive}^{1}$ (see Table 1). Data was reduced in AIPS using standard methods and total and polarization maps were constructed. We then stacked the images with a weighting based on their rms noise. Rotation measure, polarization degree and intrinsic polarization angle maps were hereafter created. We succeeded to derive robust RM maps thanks to our image stacking, wide range in the $\lambda^{2}$ space and high resolution.

\section{Results}

We show the rotation measure map in Figure 1. Values of the RM of the order of few thousands rad $\mathrm{m}^{-2}$ in HST-1 and few hundreds in knots $\mathrm{A}, \mathrm{B}$ and $\mathrm{C}$ are seen. We find, for the first time, RM gradients transverse to the M87 jet in HST-1 and knots A and C. Such RM gradient is not seen in knot B. Unlike previous works on other sources, a gradient is not detected on a single isolated component, but in various components on arc second scales of the M87 jet.

In order to investigate these gradients, we have taken slices across HST-1 and knots A, B and C (see Figure 2 ). We find the gradients in HST-1, A and C are very smooth and confirm the lack of gradient on knot B, with RM values having more irregular behavior. Gradients extend at least two beam sizes across the jet, except for

\footnotetext{
${ }^{1}$ https://archive.nrao.edu
}

the case of the HST-1 complex, which spans just about one beam size. Relative errors for the gradients, taken as $\operatorname{err}(\mathrm{RM}) /\left[\mathrm{RM}_{\max }-\mathrm{RM}_{\min }\right]$, are roughly $8 \%, 21 \%$ and $25 \%$ for HST-1 and knots A and C respectively. We find that all gradients are very robust: even for the case of HST-1, extending just one beam size, relative error is small enough to fulfill reliability criteria $[21,22,35]$.

We plot Faraday corrected magnetic vectors in Figure 1. Magnetic vectors seem to follow the direction of the jet on knot B, whereas it seems to be almost perpendicular to it in HST-1 and knots A and C. The overall trend agrees with previous results [7], although they found a small bowshock shape in knot A (i.e, magnetic vectors seem to lean in the edges of the jet, but not enough to produce a spinesheath structure). We suggest this effect is less evident in our observations as we were able to correct for a larger range of frequencies whereas they used only $6 \mathrm{~cm}$ data.

We study fractional polarization across the jet in Figure 2. There are various distinct features common to all regions studied here: fractional polarization is asymmetric with respect the centre of the jet and there seems to be an increase towards the edges, reaching up to $\sim 60 \%$. There seems to be an additional polarization component in the center of knots $\mathrm{A}$ and $\mathrm{C}$, which causes the overall fractional polarization profile across the jet in these components to be "W-shaped". This feature is not unique to knot A of M87 but has also been observed on kilo parsec scales in other sources such as $1055+018$ [26], NGC 315 [36] or $1633+382$ [22]. We note here that HST-1 is not resolved and its transverse profile might be partially smoothed by beam convolution.

\section{Magnetic fields in the M87 jet}

\subsection{Tracers of Helical Magnetic Fields}

The observation of a smooth RM gradient including a change from positive to negative values cannot be easily explained by alternative phenomena such as gradients of electron density or interaction with the media. This points towards the presence of a helical magnetic field in the M87 kilo parsec jet.

There is another interpretation for the RM in HST-1 and knots $\mathrm{A}$ and $\mathrm{C}$ : as noted by [7], there is an appreciable amount of Faraday screen after knot $\mathrm{C}$, where the jet bends and enters or blends with the lobe. This would cause contribution to the RM which would be external to the knots discussed here and not connected to them. We consider this contribution to be minimal to the RM studied here. If the RM arises from an external Faraday screen, likeliness of it being consistent with a helical magnetic field picture by chance can be estimated as follows: Let us consider for simplicity only the sign of the RM on both sides across the jet as a fiducial indicator of a gradient. In this case, probability of having a change on the RM sign on HST-1 and the same one on knots $\mathrm{A}$ and $\mathrm{C}$, but not on knot B is $p=1 / 2 \times 1 / 4^{2} \times 1 / 2<2 \%$. We note that here we did not account for gradient smoothness, which is clear in our observations. 


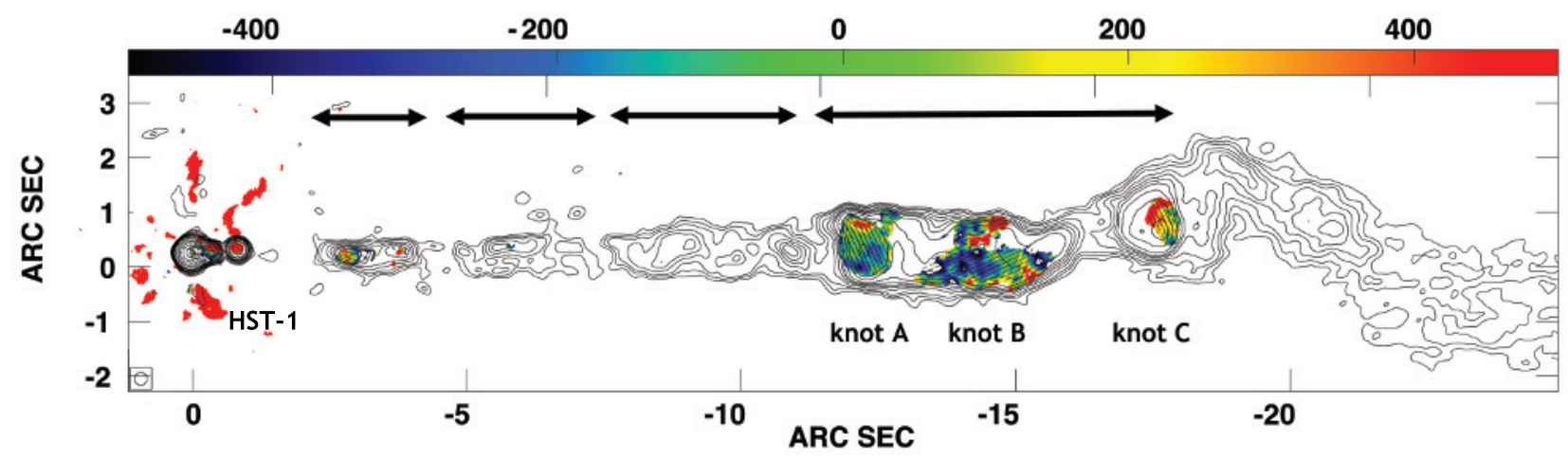

Figure 1. M87 map. Knots discussed in the text are marked. Contours indicate total intensity, with RMS $=1.13 \times 10^{-3} \times(2,3,4,6,8$, $10,12,16,24,32,64,128,256,512)$. Sticks indicate Faraday corrected magnetic vectors. Color scale indicates RM gradient from $-500 \mathrm{rad} \mathrm{m}^{-2}$ to $500 \mathrm{rad} \mathrm{m}^{-2}$. Thick arrows represent the trail of components as expected in [32] model for eye guidance.

The profile of the polarization across the jet is asymmetric, with an increase towards the edges of the jet, as expected from a helical magnetic field. [18, 29, 30]. The higher fractional polarization in the centre of the jet has also been modeled in this context [37]. Observed high degree of polarization, typically $>10 \%$ [38], even at inter knot regions of the M87, jet suggest that the scenario of a locally compressed random weak magnetic field is not the case here [32].

The most natural way to explain the combined information from i) the high fractional polarization, increasing towards the edges of the jet, ii) polarization position angles and iii) rotation measure gradients, is to assume a helical magnetic field in the jet of M87. In this picture, the magnetic structure of the jet is that of an ordered magnetic field with a three dimensional helix. As we have seen, although other models, such as a tangled magnetic field, may be able to model some properties observed in the M87 jet, cannot completely explain all of them.

\subsection{Constraints on the magnetic pitch angle}

If we assume a helical magnetic field, we can obtain a constraint on the pitch angle $\psi=\arctan \left(B_{\phi} / B_{z}\right)$ based on the RM. In general, we can decompose $B_{\text {los }}=B_{\phi} \sin \theta+$ $B_{z} \cos \theta$, where $B_{\phi}$ is the azimuthal and $B_{z}$ the axial component of the magnetic field. In order to have a smooth RM gradient with both signs for HST-1 and knots A and C, this suggests $\psi \gtrsim 75^{\circ}$ in these regions. In a similar way, the lack of a clear gradient in knot B indicates that here the poloidal component dominates and, similarly, we suggest $\psi \lesssim 75^{\circ}$ for knot B.

Magnetic vector polarization angles can also be explained in the context of a helical magnetic field. In this picture, polarization from both the rear and the front of the emitting regions are geometrically added, but polarization angles have a $180^{\circ}$ offset and the resulting observed polarization angle will depend on the pitch angle. For large (small) $\psi$ the toroidal (poloidal) component dominates and the addition will produce magnetic polarization angle perpendicular (parallel) to the jet, the threshold between these two possibilities given by $B_{\phi} / B_{z}=\sin \theta$ [39]. If we apply this model to the polarization observed in the M87 jet, we obtain $\psi \gtrsim 15^{\circ}$ for HST- 1 and knots A and C; and $\psi \lesssim 15^{\circ}$ for knot $\mathrm{B}$. This result is consistent with the one discussed from the rotation measure.

Combining the information from the rotation measure and the magnetic vectors, we find the magnetic helix is toroidally dominated, with a large pitch angle $\psi \gtrsim 75^{\circ}$ in HST-1 and knots A and C, whereas it is poloidally dominated, with a lower pitch angle $\psi \lesssim 15^{\circ}$ in knot $\mathrm{B}$. This is in agreement with the idea that knots $\mathrm{A}$ and $\mathrm{C}$ are shocked regions where the magnetic field is more compressed than in the post-shocked region of the knot B.

\subsection{A-B-C Complex as Quad MHD System}

The magnetic structure of knots A-B-C as a whole is difficult to explain with pure hydrodynamic models, unless these are taken to be totally independent features. The structure of the whole kilo parsec jet shows hints that this does not seem to be the case. Indeed, simple inspection of the total intensity shows a trail of components from HST-1 to knot C (see thick horizontal arrows in Figure 1). Magneto-hydrodynamic (MHD) models are directly able to explain this morphology together with the magnetic properties discussed above.

In this model [32], a series of MHD quad shocks are originated in the HST-1 complex and powered by torsional Alfvén waves. Thus, each of the components in the trail can be directly identified as one of these quad shocks. Under this context, knots $\mathrm{A}$ and $\mathrm{C}$ are not different shocks with their independent forward and reverse modes but twin features corresponding to the forward and reverse fast mode shocks, whereas knot B would be the post-shocked region. This MHD model predicted $\psi \sim 88^{\circ}$ on knot A, considering conical expansion of the M87 jet from HST-1. This value is in agreement with the lower limit obtained here $\psi \gtrsim 75^{\circ}$.

The shape of the jet, appearing to shrink after knot B, can also be explained in the context of a MHD model as a current-driven instability (CDI). MHD shocks can play 

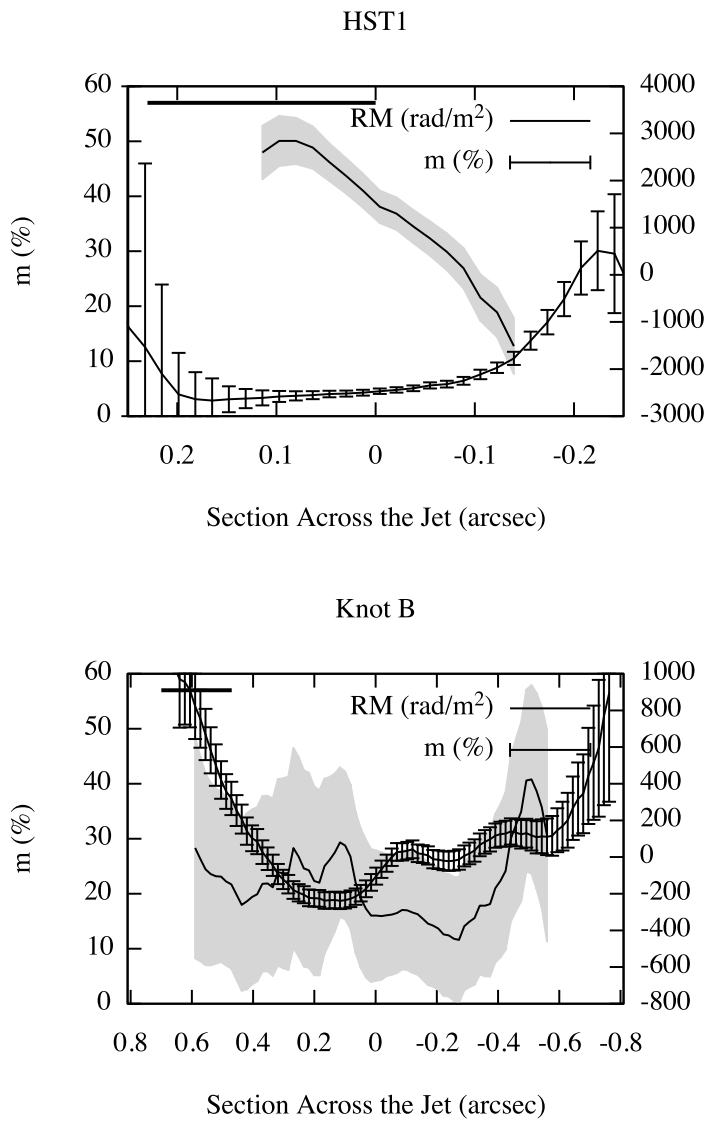
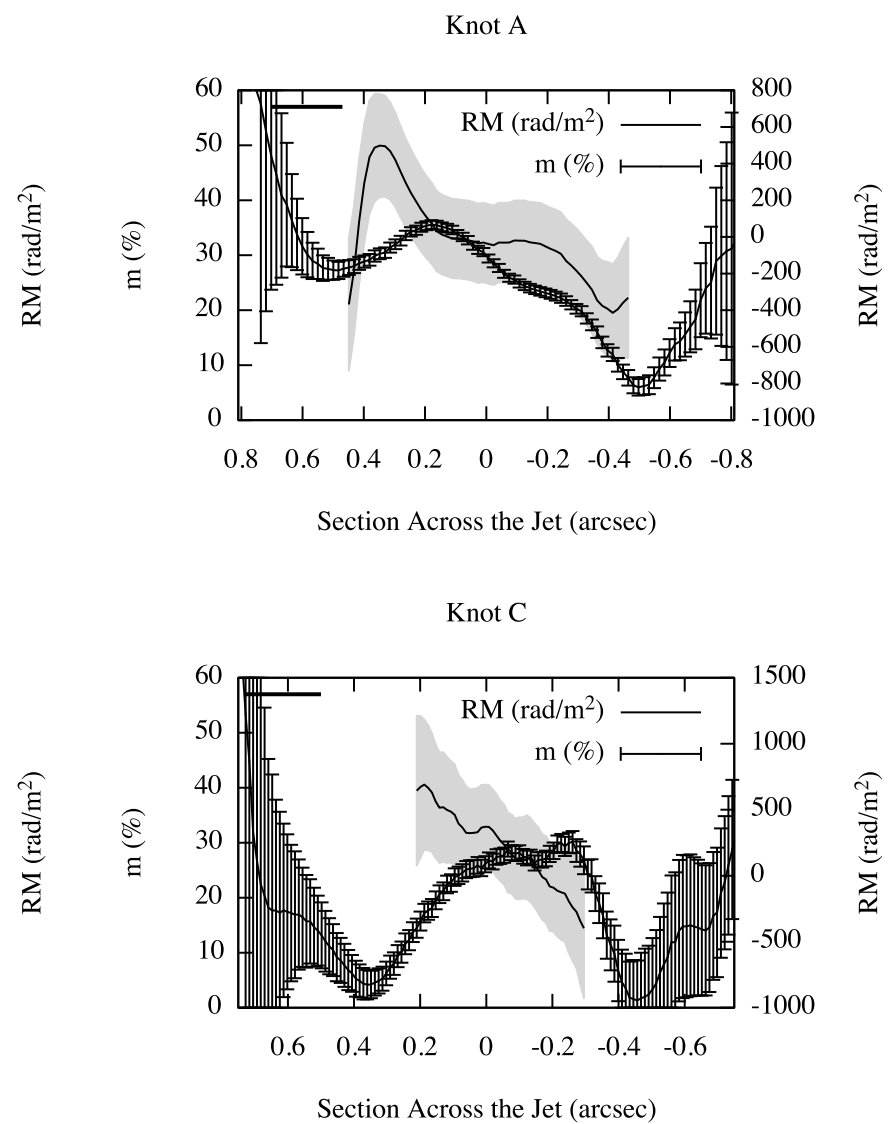

Figure 2. Slices of polarization and RM across HST-1 and knots A, B and C (from north to south). Line with black error bars indicates fractional polarization. Line with grey filling for errors indicates RM. The thick black line on the top left corner indicates the beam size, for comparison.

an important role in order to trigger these CDI [40]. We suggest that this may be the case here. The high degree of polarization, the asymmetry of the fractional polarization across the jet between knots $\mathrm{A}$ and $\mathrm{C}$ and the geometrical distortion (side-way bending from south to north) of the jet after knot B support this model. Hence, the complex form by the A-B-C knots could be a good example for the interplay between the magneto-hydrodynamic shocks and current-driven instabilities where the magnetic field may control the dynamics.

\section{Conclusions}

We have stacked a series of archival multifrequency $(8+15+22+43 \mathrm{GHz})$ polarimetric VLA observations towards M87 in order to enhance sensitivity towards the detection of robust RM gradients. Our analysis of the stacked data shows for the first time robust rotation measure gradients across various knots in the M87 jet. We detect such gradients in the HST-1 complex and knots A and C, whereas none is observed in knot B.

In these regions where the gradient is observed, it extends smoothly over more than two beam sizes and runs from positive to negative values. We discuss that probability for these gradients to arise spuriously due to contribu- tions of an external Faraday screen not related with the jet (for example, coming from the external lobes) by chance is less than $2 \%$.

Faraday-corrected magnetic vectors seem to be parallel to the jet and following its direction in knot B, whereas it seems to be perpendicular in knots A and C. High fractional polarization is found, with $m$ showing hints of an asymmetric increase towards the edges. An additional polarization component is seen in knots $\mathrm{A}$ and $\mathrm{C}$, producing an increase of the fractional polarization in the centre of these knots.

The observations from the i) RM gradients, ii) fractional polarization and iii) magnetic polarization angle can be easily interpreted in the context of a helical magnetic field. All the results presented here lead to the indication of a magnetically-dominated jet, with a well structured helical magnetic field at scales of several thousands of Schwarzschild radii along a kilo parsec from HST-1 to knot C. This is the first time evidence for such lengthy helical magnetic field is systematically seen.

Morphology and magnetic structure of the M87 kilo parsec jet can be explained as a series of MHD quad shocks launched from the HST-1 complex. This model predicts that knots $\mathrm{A}, \mathrm{B}$ and $\mathrm{C}$ are not independent structures but correspond to the fast forward, reverse and inter- 
shock regions of a quad shock, with predicted pitch angle for knot $\mathrm{A} \psi \sim 88^{\circ}$ in agreement with the lower limit found by observations $\psi \gtrsim 75^{\circ}$. The kink and jet bending after knot B can be easily interpreted under this context as a current-driven instability.

\section{Acknowledgements}

We are grateful to the ASIAA VLBI group. We also would like to thank the organizers for such successful event which lead to much discussion. The National Radio Astronomy Observatory is operated by Associated Universities, Inc., under contract with the National Science Foundation.

\section{References}

[1] Macchetto, F., Marconi, A., Axon, D., Capetti, A., Sparks, W., Crane, P., ApJ 489, 579 (1997)

[2] Gebhardt, K., Adams, J., Richstone, D., Lauer, T. R., Faber, S. M., Gültekin, K., Murphy, J., Tremaine, S., ApJ 729, 119 (2011)

[3] Tonry, J. L., ApJ 373, L1 (1991)

[4] Biretta, J. A., Sparks, W. B., Macchetto, F., ApJ 520, 621 (1999)

[5] Wang, C. C., Zhou, H. Y., MNRAS 395, 301 (2009)

[6] Owen, F. N., Hardee, P. E., Bignell, R. C., ApJ 239 , L11 (1980)

[7] Owen, F. N., Hardee, P. E., Cornwell, T. J., ApJ 340, 698 (1989)

[8] Owen, F. N., Eilek, J. A., Keel, W. C., ApJ 362, 449 (1990)

[9] Biretta, J. A., Stern, C. P., Harris, D. E., AJ 101, 1632 (1991)

[10] Perlman, E. S., Biretta, J. A., Zhou, F., Sparks, W. B., Macchetto, F.D., ApJ 117, 2185 (1999)

[11] Perlman, E. S., Biretta, J. A., Sparks, W. B., Macchetto, F.D., Leahy, J.P., ApJ 551, 206 (2001)

[12] Perlman, E. S., Adams, S. C., Cara, M., Bourque, M., Harris, D.E., Madrid, J. P., Simons, R. C., ClausenBrown, E., Cheung, C. C., Stawarz. Ł., Georganopoulos, M.,Sparks, W. B., Biretta, J. A., ApJ 347, 119 (2011)

[13] Hardee, P. E., Eilek, J. A., ApJ 735, 61 (2011)

[14] Blandford, R. D. in Astrophysical Jets, ed. D. Burgarella, M. Livio, \& C. P. O'Dea Cambridge: Cambridge Univ. Press (1993)

[15] Asada, K., Inoue, M., Uchida, Y., Kameno, S., Fujisawa, K., Iguchi, S., Mutoh, M., PASJ 54, L39 (2002)

[16] Gabuzda D. C., Murray, E., Cronin, P., MNRAS, 351, 89 (2004)
[17] Zavala, R. T. \& Taylor, G. B., ApJ 626, 73 (2005)

[18] Gómez, J. L., Marscher, A. P., Jorstad, S. G., Agudo, I., Roca-Sogorb, M., ApJL 681, L69 (2008)

[19] Mahmud, M.; Gabuzda, D. C.; Bezrukovs, V., MNRAS 400, 2 (2009)

[20] Croke, S. M., O’Sullivan, S. P., Gabuzda, D. C., MNRAS 402, 259 (2010)

[21] Hovatta, T., Lister, M. L., Aller, M. F., Aller, H. D., Homan, D. C., Kovalev, Y. Y., Pushkarev, A. B., Savolainen, T. , AJ 144, 105 (2012)

[22] Algaba, J. C., MNRAS 429, 3551 (2013)

[23] Gabuzda D. C., Cawthorne T. V., MNRAS 283, 759 (1996)

[24] Gabuzda D. C., New Astron. Rev. 43, 691 (1999)

[25] Gabuzda D. C., Pushkarev A. B., in Laing R., Blundell K., eds, ASP Conf. Ser. Vol. 250, Particles and Fields in Radio Galaxies. Astron. Soc. Pac., San Francisco, p. 180 (2002)

[26] Attridge J. M., Roberts D. H., Wardle J. F. C., ApJ 518, 87 (1999)

[27] Papageorgiou A., PhD Thesis, University of Central Lancashire (2005)

[28] Murphy, E.; Cawthorne, T. V.; Gabuzda, D. C., MNRAS 430, 1504 (2013)

[29] Clausen-Brown, E., Lyutikov, M., Kharb, P., 2011, MNRAS, 415, 2081

[30] Agudo, I., Gómez, J. L., Casadio, C., Cawthorne, T. V., Sogorb, M. R., ApJ 752, 92 (2012)

[31] Laing, R. A., MNRAS 193, 439 (1980)

[32] Nakamura, M.,Garofalo, D., Meier, D. L., ApJ 721, 1783 (2010)

[33] White, R. L., Helfand, D. J., Becker, R. H., Glikman, E., \& de Vries, W., ApJ 654, 99 (2007)

[34] Hancock, P. J., Gaensler, B. M., Murphy, T., ApJ 735, 35 (2011)

[35] Mahmud, M.; Coughlan, C. P.; Murphy, E.; Gabuzda, D. C.; Hallahan, D. R., MNRAS 43, 695 (2013)

[36] Laing, R. A.; Canvin, J. R.; Cotton, W. D.; Bridle, A. H., MNRAS 368, 48 (2006)

[37] Laing, R. A., Canvin, J. R., Bridle, A. H., in Proceedings of the International Conference: "The Origin and Evolution of Cosmic Magnetism” 2006; eds R. Beck, G. Brunetti, L. Feretti, and B. Gaensler

[38] Sparks, W. B., Biretta, J. B., Macchetto, F, ApJ, 473, 254 (1996)

[39] Asada, K., Inoue, M., Nakamura, M., Kameno, S., Nagai, H., ApJ 682, 798 (2008)

[40] Nakamura, M., Meier, D. L., ApJ 617, 123 (2004) 